\title{
Ubiquitous Induction of p53 in Tumor Cells by Antisense Inhibition of MDM2 Expression
}

\author{
Lihong Chen,' Wenge Lu, ${ }^{1}$ Sudhir Agrawal, ${ }^{2}$ Wenqiang Zhou, \\ Ruiwen Zhang, ${ }^{3}$ and Jiandong Chen ${ }^{1}$ \\ ${ }^{1}$ Department of Microbiology, Stanley S. Scott Cancer Center, Louisiana \\ State University Medical Center, New Orleans, Louisiana, U.S.A. \\ ${ }^{2}$ Hybridon, Inc., Cambridge, Massachusetts, U.S.A. \\ ${ }^{3}$ Division of Clinical Pharmacology, University of Alabama at \\ Birmingham, Birmingham, Alabama, U.S.A.
}

Accepted December 11, 1998.

\begin{abstract}
Background: The $M D M 2$ oncogene functions as a negative feedback regulator of the p53 tumor suppressor. Abnormal expression of MDM2 in tumors may attenuate the p53-mediated growth arrest and apoptosis response, resulting in increased cell proliferation and resistance to chemotherapy.

Materials and Methods: We have developed phosphorothioate antisense oligodeoxynucleotides optimized for inhibition of MDM2 expression and investigated the role of MDM2 in a large panel of tumor cell lines.

Results: Inhibition of MDM2 expression in 15 tumor types containing wild-type p53 results in a significant induction of nuclear $\mathrm{p} 53$ accumulation. The increase in

p53 level is due to prolonged half-life and is associated with an increase in p53 transcriptional activity, growth inhibition, or apoptosis. Inhibition of MDM2 expression is also sufficient to induce nuclear p53 accumulation in several cell lines with cytoplasmic p53.

Conclusions: The MDM2 negative feedback loop is important for maintenance of $\mathrm{p} 53$ at a low level by promoting p53 degradation. Nuclear export and degradation by MDM 2 may contribute to the p53 nuclear exclusion phenotype. Inhibition of MDM2 expression can effectively activate p53 in most tumor types, including those without MDM2 overexpression, and may have broad anti-tumor potential.
\end{abstract}

\section{Introduction}

The $p 53$ tumor suppressor gene functions through induction of growth arrest or apoptosis in response to stress or genomic damage (1). About $50 \%$ of tumors carry p53 mutations (2), suggesting that presence of a functional p53 pathway is incompatible with tumor growth. Tumors without p53 mutation may contain an impaired p53 pathway due to alternative mechanisms, such as overexpression of the MDM2

Address correspondence and reprint requests to: Dr. Jiandong Chen, Department of Microbiology, Stanley S. Scott Cancer Center, Louisiana State University Medical Center, 1901 Perdido Street, New Orleans, LA 70112, U.S.A. Phone: 504-568-5608; Fax: 504-568-2918; E-mail: jchen@lsumc.edu oncogene $(3,4)$. p5 3 is an important mediator for the anti-cancer effects of many chemotherapeutic agents and ionizing irradiation (5). Therefore, development of methods to activate p53 in tumor cells will shed light on the mechanism of p53 regulation and provide a potential new anticancer strategy.

The $M D M 2$ oncogene was first cloned as an amplified gene on a murine double-minute chromosome (6). Overexpression of MDM2 in cells increases the tumorigenic potential (6) and can cooperate with an activated ras oncogene to attain full transformation (7). The MDM2 gene is amplified or overexpressed in about $40-60 \%$ of human osteogenic sarcomas and about $30 \%$ of 
soft-tissue sarcomas $(3,4)$, implicating its role in the development of these malignancies.

An important function of MDM2 is to bind to and inhibit the $\mathrm{p} 53$ tumor suppressor (8). MDM2 can also promote degradation of $\mathrm{p} 53$ through a ubiquitin-dependent proteolytic pathway by acting as a ubiquitin ligase E3 $(9-11)$. p53 activates MDM2 expression at the level of transcription $(12,13)$, suggesting that MDM2 can function as a negative feedback regulator of $\mathrm{p} 53$. Experiments using MDM2 knockout mice confirmed that an important function of MDM2 is to negatively regulate p53 $(14,15)$. Recently, it was demonstrated that introduction of antibody $3 G 5$ (16), or an engineered protein that can inhibit MDM2p55 binding, resulted in stimulation of $p 53$ activity and accumulation of p53 in MCF-7 and T22 cells (17). Therefore, MDM2 may have a constitutive role in promoting $\mathrm{p} 53$ turnover.

We previously identified a phosphorothioate antisense oligonucleotide against human $M D M 2$. Inhibition of MDM2 expression in a tumor cell line (JAR) with MDM2 amplification resulted in activation of $\mathrm{p} 53$ and apoptosis. The MDM2 antisense oligonucleotide also enhanced the ability of a DNA damaging agent (camptothecin) to induce p53 in a synergistic fashion (18), suggesting that the MDM2 feedback loop may limit the magnitude of $\mathrm{p} 53$ activation during a DNA-damage response. Therefore, inhibition of MDM2 expression may have therapeutic potential against human tumors expressing wild-type p53. However, many questions remain to be addressed, i.e., the effects of inhibiting MDM2 expression in tumors with different $\mathrm{p} 53$ mutation status, level, localization, stability, and MDM2 level. Furthermore, oligonucleotides identified in a primary screen is unlikely to be optimal.

In this study, we have developed a significantly improved MDM2 antisense phosphorothioate oligodeoxynucleotide that is a potent activator of $\mathrm{p} 53$. Inhibition of MDM2 expression in a large panel of human tumor cell lines using this oligonucleotide revealed that MDM2 is widely involved in suppressing the accumulation of $\mathrm{p} 53$. Therefore, inhibition of MDM2 expression is a powerful method for activating $\mathrm{p} 53$ by inducing $\mathrm{p} 53$ accumulation in the absence of DNA damage.

\section{Materials and Methods}

Synthesis of Oligodeoxynucleotides

Phosphorothioate oligodeoxynucleotides were synthesized using $\beta$-cyanoethyl phosphoramid- ite chemistry on an automated synthesizer (Expedite 8909, Perceptive Biosystems, Framingham, MA) and purified by preparative reversephase high-performance liquid chromatography. Seven 20-mer phosphorothioate antisense oligodeoxynucleotides (AS1-AS7) were synthesized for this screen (Fig. 1). A 2-bp mismatch control (AS2M2: 5'TGACACTTGTTCTTACTCAC) and a 4-bp mismatch control oligodeoxynucleotide (AS2M4: 5'TGACTCTTGTCCTTACTCAC) for AS2 were also synthesized. The oligodeoxynucleotide $\mathrm{K}$ (5'CAGAGCCTTCATCTTCCCAG) is a control against an unrelated target.

\section{Cell Lines}

JAR, JEG-3, SJSA, MCF-7, U87-MG, SK-N-SH, U2OS, Caski, C33A, DLD-1, and A549 cells were obtained from the ATCC. WI-38, JeKin, HepG2, LS180, HT1080, G361, PA-1, and Lncap cells were obtained from the cell culture core lab of LSU Medical Center. H1299, MCF-7, MDA-MB231, and Hela cells were from Dr. Arnold J. Levine (Princeton University). The 101 cell line was provided by Dr. James Gnarra (LSU Medical Center). The SLK cell line was kindly provided by Dr. Om Prakash (Ochsner Foundation). All cells were grown in Dulbecco's modified Eagle's medium (DMEM) with 10\% fetal bovine serum (FBS).

\section{Antisense Oligonucleotide Treatment}

Cells were cultured in DMEM medium with $10 \%$ FBS. Cell lines normally grown in other types of medium were also adapted to growth in DMEM with $10 \%$ FBS before the treatment. Before addition of oligonucleotides, cells were refed with DMEM containing $1 \%$ FBS. Lipofectin (Gibco BRL) was incubated with serum-free DMEM medium for $45 \mathrm{~min}$, then mixed with the oligonucleotides for $10 \mathrm{~min}$ and added to the cell culture. The final concentration of Lipofectin was $7 \mu \mathrm{g} /$ $\mathrm{ml}$, final concentration of FBS was $0.75 \%$. Cells were incubated with oligonucleotides and Lipofectin for $18-24 \mathrm{hr}$ as indicated.

\section{Western Blot}

Cells were lysed in RIPA buffer $(50 \mathrm{mM}$ Tris, $\mathrm{pH}$ 7.4, $150 \mathrm{mM} \mathrm{NaCl}, 1 \%$ Triton X-100, $0.1 \%$ SDS, $1 \% \mathrm{Na}$ deoxycholate) and $100 \mu \mathrm{g}$ of the protein lysate were fractionated by SDS-polyacrylamide gel electrophoresis (SDS-PAGE) and transferred to Immobilon $\mathrm{P}$ filters (Millipore). The filters 
A

Human MDM2 non-coding strand

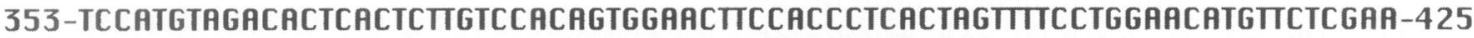

AS 1: TGTAGACACTCACTCTIGTC AS:GGAACTCСACССTCACTAG

AS2:CACTCACTCTTGTCCACAG AS5: ACСCTCACTAGTITTCCTGG

T AS3: ACTCTTGTCCACAGTGGAAC AS6: СACTAGTTITCCTGGAACAT

AS4: TGTCCACAGTGGAACTCCA AS7: ПССTGGAACATGTCTCGA

B

C

MCF7-BP100-Iuc

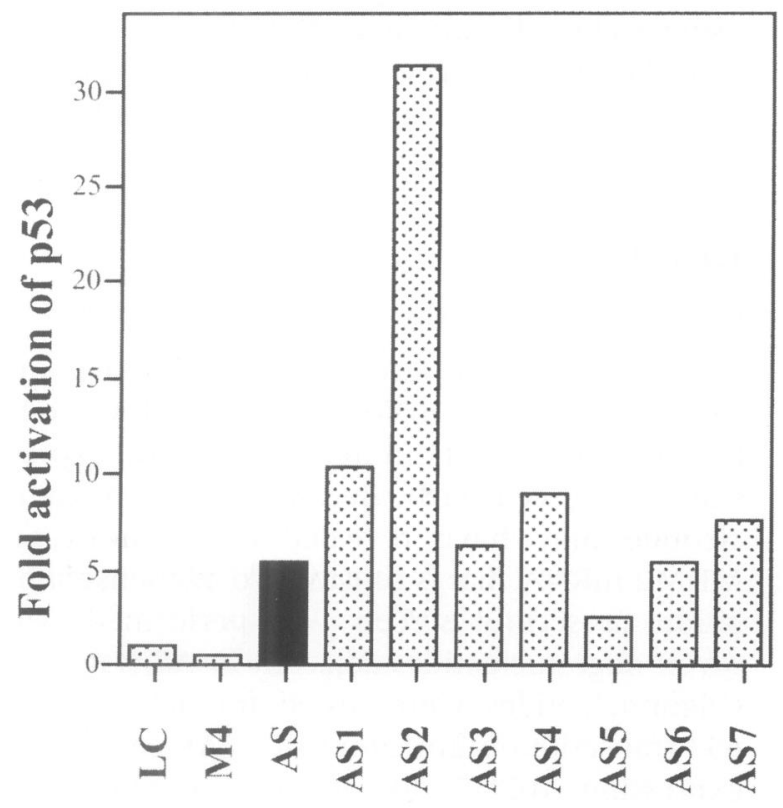

Oligonucleotides

D

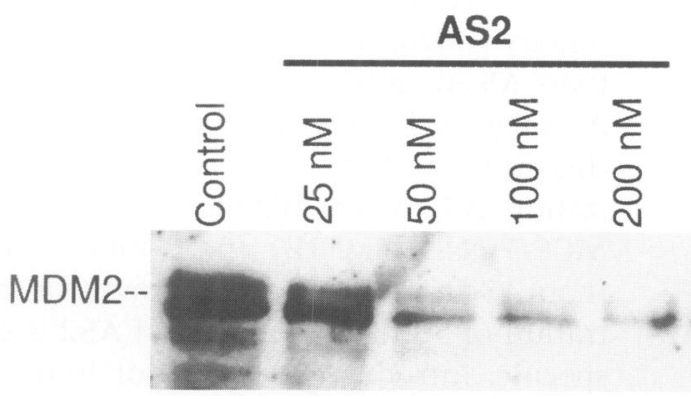

Fig. 1. Characterization of MDM2 antisense oligonucleotides. (A) Sequence of $M D M 2$ antisense phosphorothioate oligonucleotides. The top sequence represents the noncoding strand of human MDM2. The numbers indicate distance from the translation initiation codon. (B) Screening of MDM2 antisense oligonucleotides. MCF-7 cells stably transfected with the BP100-luc reporter were treated with $50 \mathrm{nM}$ of MDM2 oligonucleotides for $20 \mathrm{hr}$. p53 transcriptional activity was determined as luciferase activity/unit protein. M4 is a 4-bp mismatch control of AS. LC, control with lipofection alone. (C) Activation of p53
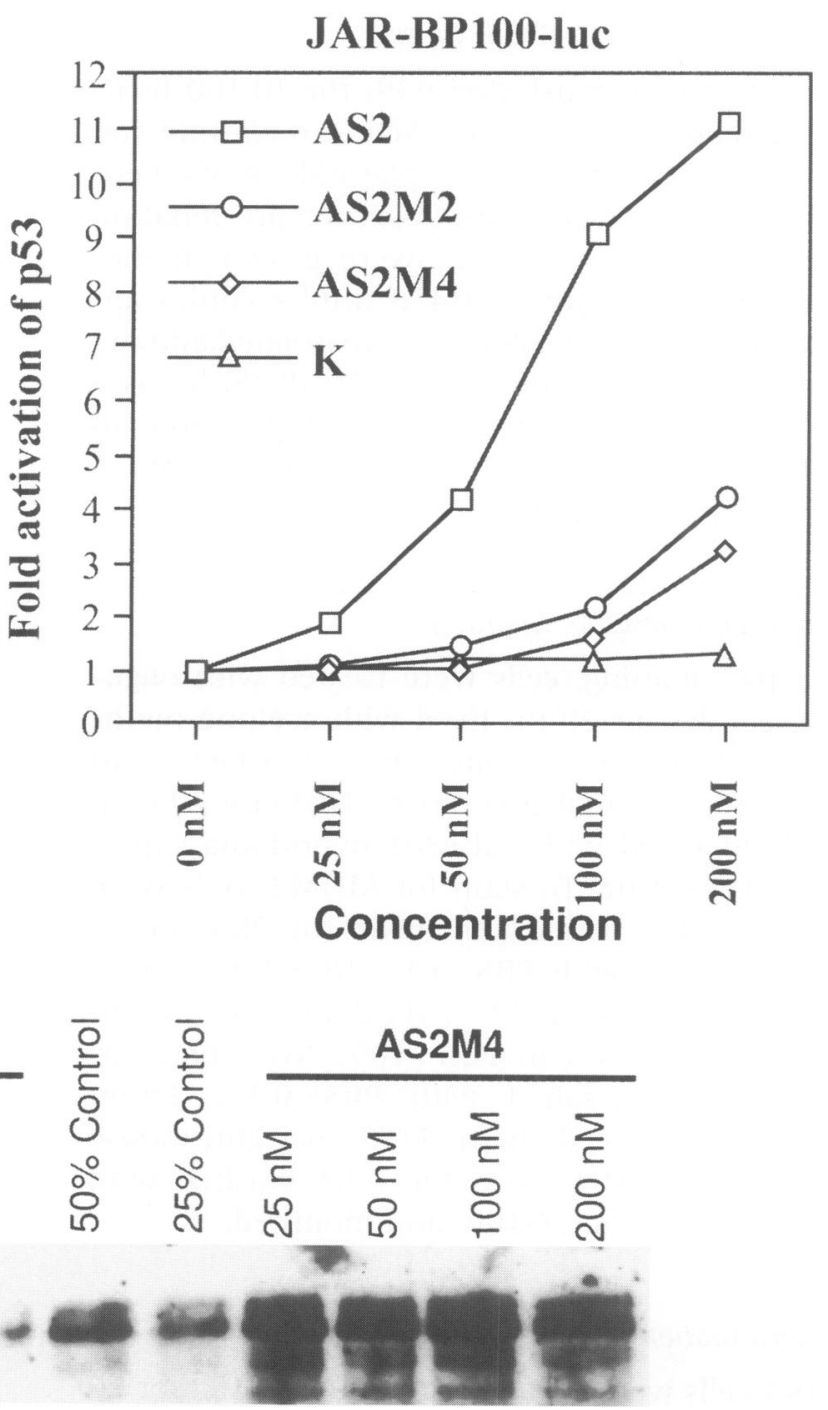

by AS2 is sequence-specific. JAR cells stably transfected with the BP100-luc reporter were treated with AS2, mismatch control oligonucleotides of AS2 (AS2M2, 2-bp mismatch; AS2M4, 4-bp mismatch), and an unrelated oligonucleotide K. (D) Inhibition of MDM2 expression by AS2. MDM2 levels in JAR cells treated for $20 \mathrm{hr}$ with AS2 or AS2M4 control oligonucleotide were determined by Western blotting using monoclonal antibody 3 G9. JAR cells treated with lipofectin alone, loaded at $1-, 2-$, and 4-fold dilutions, were used as controls. 
were blocked for $5 \mathrm{~min}$ with $5 \%$ non-fat dry milk, incubated with anti-p53 monoclonal antibody DO- 1 or an anti-MDM 2 monoclonal antibody $3 G 9$ (19), then incubated with protein Aperoxidase (Promega), washed, and developed using the ECL-plus reagent (Amersham). All incubations were carried out in phosphate-buffered saline (PBS) with 5\% non-fat milk and $0.1 \%$ Tween-20.

\section{Stable Transfection of Cell Lines}

Cells were co-transfected with the BP100-luciferase reporter plasmid or pActin-E6 plasmid and a G418-resistant marker plasmid pCMV-neoBam using the calcium-phosphate precipitation method. Transfected cells were grown in medium with $750 \mu \mathrm{g} / \mathrm{ml}$ G418 until colonies appeared. Individual colonies were isolated and expanded into cell lines. The JAR-BP100-luc is a clonal cell line isolated by diluting a pool of BP100-luciferase-transfected JAR cells from a previous experiment (18).

\section{Immunofluorescence Staining}

For p53 staining, cells were treated with oligonucleotides for $20 \mathrm{hr}$, fixed with acetone-methanol (1:1) for $3 \mathrm{~min}$, then blocked with PBS $+10 \%$ normal goat serum (NGS) for $20 \mathrm{~min}$, and incubated with Pabl801 hybridoma supernatant for $2 \mathrm{hr}$. To stain for MDM2, cells were fixed in $4 \%$ paraformaldehyde in PBS for 20 min, blocked with PBS $+10 \%$ NGS for 20 min, and incubated with $2 \mathrm{~A} 9$ hybridoma supernatant at $1 / 100$ dilution in PBS $+10 \%$ NGS for $2 \mathrm{hr}$. Slides were washed with $\mathrm{PBS}+0.1 \%$ Triton $\mathrm{X}-100$, incubated with FITC-goat-anti-mouse IgG in PBS $+10 \%$ NGS for $1 \mathrm{hr}$, washed with PBS $+0.1 \%$ TritonX-100, and mounted.

\section{Determination of $p 53$ Half-Life}

SJSA cells were treated with 200 nM AS2 for 20 hr. Cells were incubated with DMEM (without methionine) with $2 \%$ dialyzed FBS, $50 \mathrm{uCi} / \mathrm{ml}$ 35S-EXPRESS (NEN) for $2 \mathrm{hr}$ and refed with regular medium. Sample plates were collected at indicated time points and lysed with lysis buffer (50 mM Tris, pH 8.0, 5 mM EDTA, $150 \mathrm{mM} \mathrm{NaCl}$, $0.5 \%$ NP40, $1 \mathrm{mM}$ PMSF). Cell lysates with identical levels of radioactivity $\left(\sim 2 \times 10^{7} \mathrm{CPM}\right)$ were immunoprecipitated with Pab421 and Pabl801, washed with $50 \%$ SNNTE buffer $(25 \mathrm{mM}$ Tris, pH 7.4, 2.5 mM EDTA, $2.5 \%$ sucrose, $1 \%$ NP-40,
$250 \mathrm{mM} \mathrm{NaCl}$ ), and fractionated by SDS-PAGE. P53 was detected by autoradiography.

\section{Determination of Cell Proliferation Rate}

Cells were treated with $100 \mathrm{nM}$ of oligonucleotides for $20 \mathrm{hr}$, labeled with BrdU for $2 \mathrm{hr}$, and incubated with MTS reagent (Promega) for $1 \mathrm{hr}$. Relative cell viability was determined by measuring OD at $490 \mathrm{~nm}$ (reduction of MTS substrate by mitochondria activity). Cells were then fixed and the level of BrdU incorporation was determined using a chemiluminscence ELISA assay (Boehringer Mannheim). The rate of DNA synthesis was determined as BrdU incorporation/OD 490.

\section{Results}

\section{Optimization of MDM2 Antisense Oligonucleotide}

In a previous study, a screen using nine oligonucleotides identified HDMAS5 ("AS" below) to be the most effective in inhibition of MDM2 expression in JAR cells (18). Because the AS oligonucleotide may have revealed a region of the MDM2 mRNA that is sensitive to antisense inhibition, a second screen was performed with seven oligonucleotides adjacent to AS (Fig. 1A). Oligonucleotides were tested for activation of p53 transcription function using JAR cells (overexpressing MDM2 due to gene amplification) and MCF-7 cells (expressing lower levels of MDM2) stably transfected with the p53-responsive reporter plasmid BP100-luciferase.

The results showed that several of the secondary oligonucleotides were more effective than AS in causing activation of p53 (Fig. 1B). An oligonucleotide AS2 was the most potent of this group and was chosen for further characterization. AS2 was 5-fold more efficient than AS in MCF-7 cells (Fig. 1B) and 2-fold more efficient than AS in JAR cells (not shown) at a concentration of $50 \mathrm{nM}$. The effect of AS2 is sequencespecific. Introduction of two or four nucleotide mismatches into the sequence significantly inhibited its ability to activate p53 (Fig. 1C). Treatment of JAR cells with AS2 inhibited MDM2 protein expression in a sequence-dependent and dose-dependent fashion (Fig. ID), suggesting that its ability to activate p53 is due to specific inhibition of MDM2 expression. Consistent with its increased ability to activate p53, AS2 is more efficient in inhibiting MDM2 expression (IC50: $\sim 25 \mathrm{nM}$, Fig. ID) than AS (IC50: 100-200 nM) (18) in JAR cells. When AS2 or AS (20 nucleo- 
A

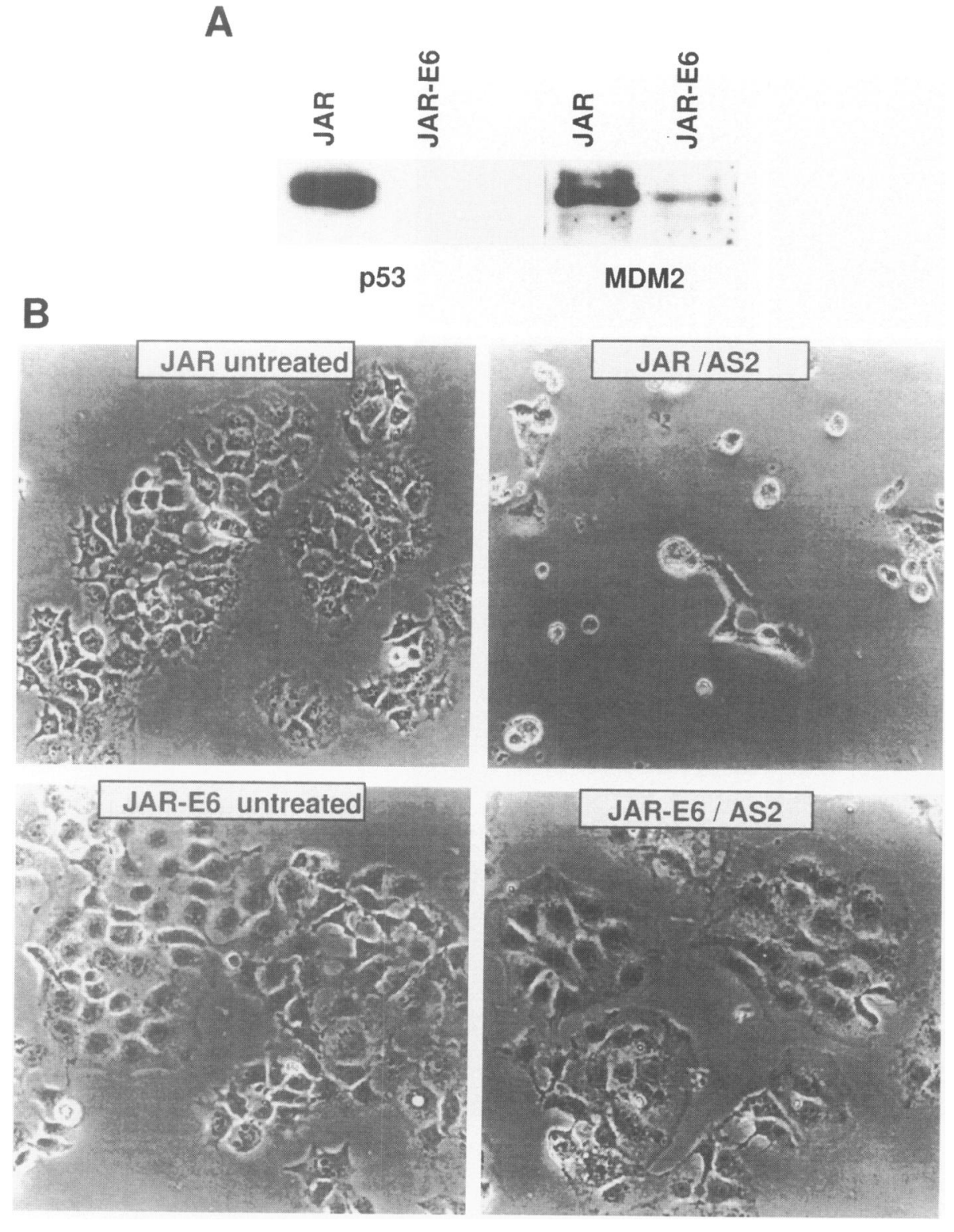

Fig. 2. Induction of apoptosis by AS2 is p53dependent. (A) JAR cells stably transfected with an actin promoter-driven HPV E6 construct (JAR-E6) expressed no detectable $\mathrm{p} 53$ and significantly reduced level of MDM2 in Western blot analyses. Identical amounts of total protein were loaded on each lane. (B) JAR-E6 cells are resistant to apoptosis induction by AS2. JAR and JAR-E6 cells were treated with 200 nM of AS2 for 24 hr. AS2 induced significant cell death in JAR cells, but not in JAR-E6 cells. tides) were shortened to 18 nucleotides from one or both ends, the ability to activate p53 was also significantly reduced (not shown).

\section{Apoptotic Function of AS2 Is p53-Dependent}

Similar to the parent AS oligonucleotide, AS2 also induces apoptosis in JAR cells. To further delineate whether AS2 induces apoptosis through activation of p53, a JAR cell line expressing the E6 oncogene of HPV16 was created (JAR-E6). Expression of E6 under the actin promoter results in degradation of $\mathrm{p} 53$, as demonstrated by the loss of $\mathrm{p} 53$ protein in a Western blot (Fig. 2). Interestingly, the level of
MDM2 expression also decreases significantly in JAR-E6 cells, suggesting that in addition to gene amplification, activation by $\mathrm{p} 53$ is an important mechanism of MDM2 overexpression in this cell line.

When treated with $200 \mathrm{nM}$ of AS2, which efficiently induced apoptosis in parental JAR cells, JAR-E6 cells showed little apoptosis (Fig. 2). This result suggests that AS2 induces apoptosis through specific activation of $\mathrm{p} 53$. The JAR-E6 cells are not resistant to apoptosis in general; treatment with DNA-damaging agents (camptothecin, etoposide) at concentrations that can kill other p53-null cells also induced cell death in JAR-E6 (not shown). 


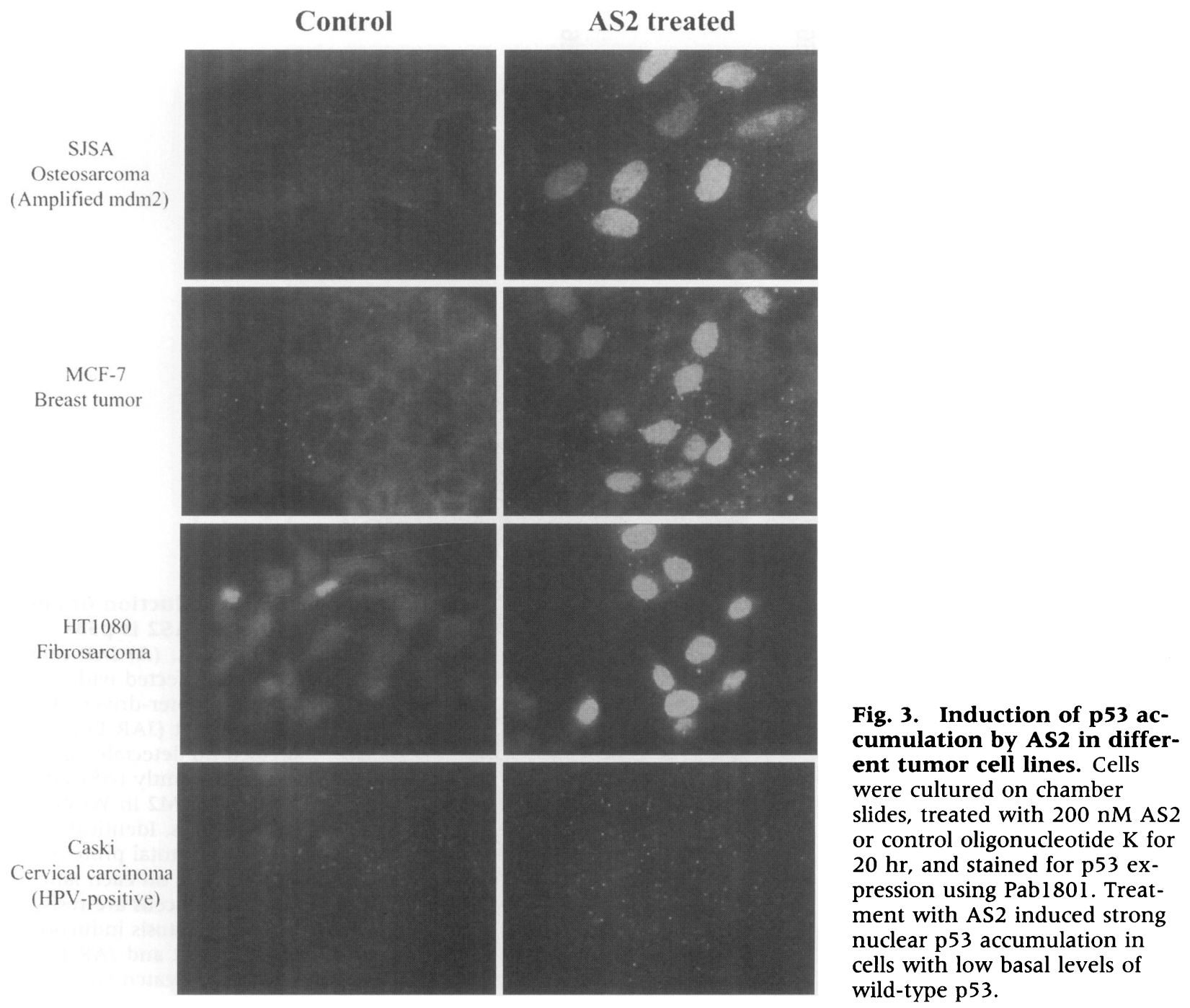

Induction of p53 Accumulation by Inhibition of MDM2 in Different Cell Lines

The strong activation of $\mathrm{p} 53$ in MCF- 7 cells by AS2 (Fig. 1) prompted us to further examine its effect on p53. MCF-7 cells predominantly contain a cytoplasmic form of p53 (20) and displayed a predominantly cytoplasmic fluorescence when stained using anti-p53 monoclonal antibody Pab1801. After treatment with $200 \mathrm{nM}$ AS2 for $20 \mathrm{hr}$, many MCF-7 cells show intense nuclear p53 staining (Fig. 3). The parent AS oligonucleotide also showed a similar but weaker ability to induce p53 accumulation (not shown), and the control oligonucleotide $\mathrm{K}$ did not induce p53 (Fig. 3). This suggests that MDM2 plays an important role in inhibiting the accumulation of nuclear p53 in this cell line. Because there is an increase in total p53 after AS2 treatment (see below in Fig. 4), and there is not an obvious loss of cytoplasmic p53 staining in cells accumulating nuclear $\mathrm{p} 53$, the results suggest that MDM2 normally promotes the degradation of nuclear p53 in MCF-7 cells.

To determine whether MDM2 also exhibits a similar role in other tumors containing cytoplasmic p53, the neuroblastoma cell line SK-N-SH was tested. Neuroblastomas rarely have p $53 \mathrm{mu}-$ tations but often contain p53 in the cytoplasm. SK-N-SH cells express cytoplasmic wild-type p53 and exhibit a reduced ability to undergo cell cycle arrest after DNA damage $(21,22)$. When treated with AS2, this cell line also displayed a strong accumulation of nuclear p53 in nearly $100 \%$ of the cells, as well as an increase in total p53 level (not shown). This result suggests that in addition to cytoplasmic sequestration, MDM2- 
A

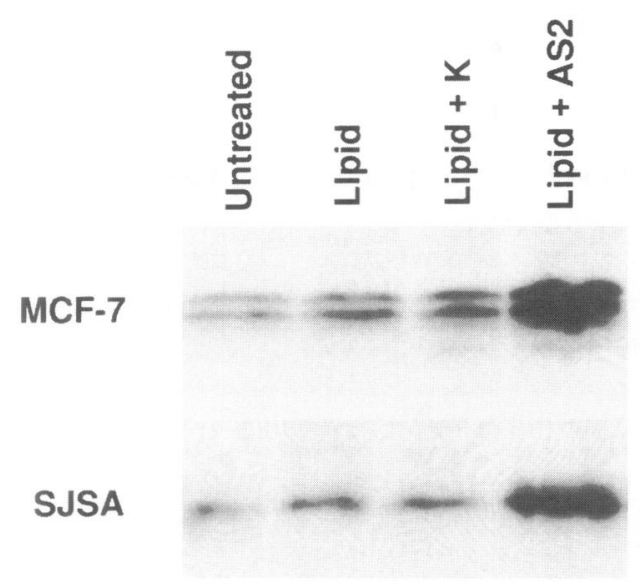

B

AS2 treated

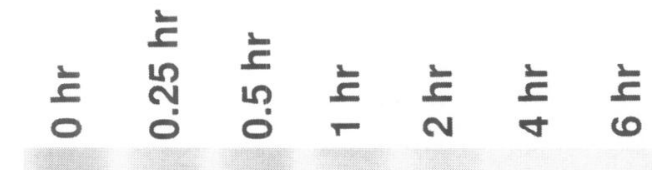

Untreated

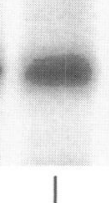

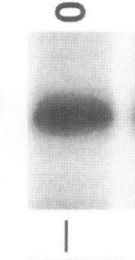

.
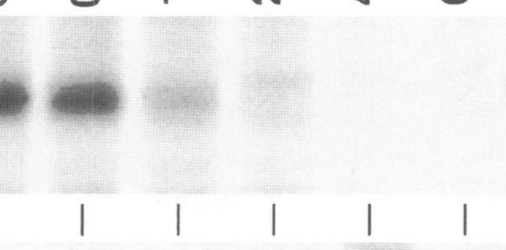

$--p 53$

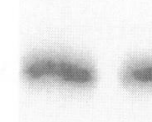

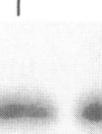
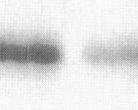

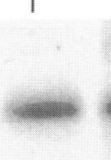

\section{.}

Fig. 4. Stabilization of p53 by inhibition of MDM2 expression. (A) Inhibition of MDM2 expression results in an increase of p53 level. Cells were treated with $200 \mathrm{nM}$ of AS2 or control oligonucleotide $\mathrm{K}$ for $20 \mathrm{hr}$. p53 protein levels were detected by Western blot with antibody DO-1. Identical amounts of total protein were loaded on each lane. The double band in MCF-7 is due to a p53 polymorphic allele. (B) Determination of p53 half-life. SJSA cells were treated with $200 \mathrm{nM}$ AS2 for $20 \mathrm{hr}$ and the rate of p53 degradation was determined by a pulse-chase experiment. The half-life of p53 was $\sim 0.5 \mathrm{hr}$ in untreated SJSA cells and $>4 \mathrm{hr}$ in AS2 treated cells as determined by densitometric analysis. The total CPM used for untreated samples was 2-fold higher than for AS2-treated samples, thus the p53 band in $0 \mathrm{hr}$ untreated sample is darker and does not indicate a different rate of synthesis. mediated degradation may be an important mechanism for the loss of nuclear p53 in some tumors (also see Discussion).

This test was then extended to a wide variety of tumor cell lines with wild-type p53. A total of 24 human tumor cell lines representing 15 different tumor types were treated with AS2 and stained for $\mathrm{p} 53$ expression. The results, as shown in Figure 3 and summarized in Table 1, revealed that the low levels of wild-type p53 can be significantly stimulated by AS2, resulting in intense nuclear p53 staining. Two nontransformed human cell lines, WI-38 (lung fibroblast) and JeKin (skin fibroblast), also showed strong p53 accumulation after inhibition of MDM2 expression. Therefore, this p53 response to inhibition of MDM2 expression is not unique to tumor cells.

Treatment of tumor cells with homozygous mutant p53 also did not lead to further accumulation of p53, which was already at a high level. A tumor cell line with both wild-type and mutant p53 alleles (PA-1) also contains inducible p53 and underwent apoptosis after AS2 treatment (see below). Finally, treatment of HPV- positive cervical cancer cell lines did not induce p53 accumulation, suggesting that HPV E6-mediated degradation of $\mathrm{p} 53$ is independent of MDM2 function.

\section{Inhibition of MDM2 Expression Prolonged p53 Half-Life}

The increase in p53 after inhibition of MDM2 expression can result from an increased rate of p53 synthesis or protein stabilization. To directly test these possibilities, the p53 half-life in AS2treated SJSA cells was determined by a pulsechase radioactive labeling experiment. SJSA cells have $M D M 2$ gene amplification and exhibit a highly inducible wild-type p53 after AS2 treatment (Figs. 3, 4A). This cell line does not undergo significant apoptosis after AS2 treatment, thus it can provide sufficient material for analysis.

SJSA cells were treated with 200 nM AS2 for $20 \mathrm{hr}$ and pulse labeled with ${ }^{35} \mathrm{~S}$-methionine for $2 \mathrm{hr}$. The level of p53 was determined at various times after addition of excess cold methionine to 


\section{Table 1. Induction of p53 accumulation by inhibition of mdm2 expression ${ }^{a}$}

\begin{tabular}{|c|c|c|c|c|c|}
\hline \multirow[b]{2}{*}{ Cell Line } & \multirow[b]{2}{*}{ Origin } & \multirow[b]{2}{*}{ MDM2 Level } & \multirow[b]{2}{*}{ p53 } & \multicolumn{2}{|c|}{ Nuclear p53 Level } \\
\hline & & & & Basal & AS2 Treated \\
\hline JEG-3 & Choriocarcinoma & ++++ & wt & ++++ & ++++ \\
\hline JAR & Choriocarcinoma & ++++ & wt & ++++ & ++++ \\
\hline SJSA & Osteosarcoma & +++ & wt & - & ++++ \\
\hline LS 180 & Colon carcinoma & - & wt & - & ++++ \\
\hline HT1080 & Fibrosarcoma & + & $w t$ & + & ++++ \\
\hline Al72 & Glioblastoma & - & wt & - & ++++ \\
\hline U87-MG & Glioblastoma & - & $\mathrm{wt}$ & + & ++++ \\
\hline HepG2 & Hepatocarcinoma & + & wt & + & ++++ \\
\hline SLK & Kaposi's sarcoma & - & ND & + & ++++ \\
\hline 101 & Kidney tumor & - & wt & + & ++++ \\
\hline A549 & Lung tumor & + & wt & + & ++++ \\
\hline G361 & Melanoma & ++ & wt & + & ++++ \\
\hline SK-N-SH & Neuroblastoma & - & wt & - & ++++ \\
\hline MCF-7 & Breast carcinoma & + & wt & + & ++++ \\
\hline U2OS & Osteosarcoma & + & wt & ++ & +++ \\
\hline PA-1 & Ovarian teratoma & + & $\mathrm{wt} / \mathrm{mt}$ & - & ++++ \\
\hline Lncap & Prostate carcinoma & - & $\mathrm{wt}$ & + & ++++ \\
\hline WI-38 & Lung fibroblast & + & wt & - & ++++ \\
\hline JeKin & Skin fibroblast & - & wt & + & +++ \\
\hline MDA-MB-231 & Breast carcinoma & ++ & $\mathrm{mt}$ & ++++ & ++++ \\
\hline DLD-1 & Colon carcinoma & ++ & $\mathrm{mt}$ & ++++ & ++++ \\
\hline C33A (HPV-) & Cervical carcinoma & ++ & $\mathrm{mt}$ & ++++ & ++++ \\
\hline Hela (HPV +) & Cervical carcinoma & - & wt & - & - \\
\hline Caski $(\mathrm{HPV}+)$ & Cervical carcinoma & - & wt & - & - \\
\hline H1299 & Lung tumor & - & Null & - & - \\
\hline SK-N-MC & Neuroblastoma & - & Null & - & - \\
\hline
\end{tabular}

${ }^{a}$ Cells were treated with $200 \mathrm{nM}$ of AS2 or K oligonucleotide for $20 \mathrm{hr}$ and p53 level was determined semi-quantitatively by immunofluorescence staining with Pab1801. Mdm2 levels in untreated cells were determined by staining with 2A9.

- , not detectable; + , weak staining in most cells or a subset of cells; ++ , moderate staining in most cells or a subset of cells; ++++ , strong staining in most or all cells; mt, mutant; ND, not determined; wt, wild type. Cell lines without MDM2 IF signal have been confirmed to express low levels of MDM2 by Western blot.

prevent further synthesis of radioactive $\mathrm{p} 53$. The result showed that the half-life of p53 is increased from $\sim 0.5 \mathrm{hr}$ in untreated SJSA cells to $>4 \mathrm{hr}$ in AS2-treated cells. Furthermore, the amount of radioactive MDM2 synthesized during the 2-hr pulse-labeling period did not differ significantly in treated and untreated cells, as revealed by the levels of MDM2 at time zero of the chase (Figure 4B). Therefore, the rise in p53 level after inhibition of MDM2 expression appears to be due to the stabilization of $\mathrm{p} 53$, and not to its increased synthesis.

\section{Inhibition of MDM2 Expression Induced Functional $p 53$}

To further determine whether the p53 protein that accumulates after inhibition of $M D M 2$ is functionally active, a p53-responsive BP100-luciferase reporter plasmid was transfected into 


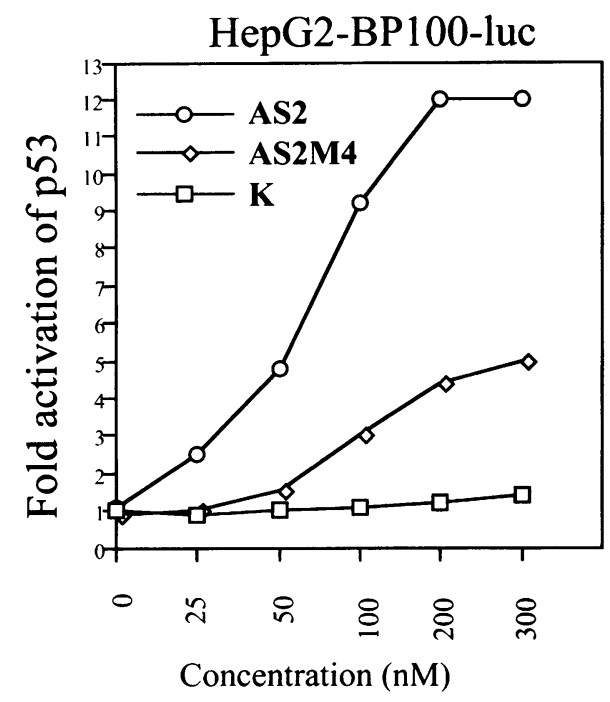

several representative cell lines. Stably transfected cells were then treated with AS2 or control oligonucleotides. The results showed that a strong induction of p53 transcription function occurs after inhibition of $M D M 2$, demonstrating that the p53 accumulated after inhibition of MDM2 is highly active (Fig. 5). The magnitude of p53 transcription activation is consistent with the fact that most of the p53 accumulation occurs in the nucleus, which has a low basal level of p53. The 4-bp mismatch control oligonucleotide has significantly reduced efficiency in activation of p53, particularly at low concentrations.

\section{Inhibition of MDM2 Leads to Growth Arrest and Apoptosis}

After a 20-hr treatment with 200 nM AS2, a significant amount of cell death was evident in some of the tumor cell lines tested (HT1080, PA-1, SK-N-SH; Fig. 6). In these cases, cells rounded up, displayed membrane ruffling and blebbing characteristic of apoptosis, and detached from the culture surface. Staining of nuclear DNA also showed shrinkage and fragmentation of nuclei (Fig. 6B). Thus, it appears that the level of p53 activation achieved by treatment with AS2 can be sufficient to induce cell death through apoptosis in some cell lines examined.

Some of the tumor cell lines (MCF-7, SJSA, A549, A174) as well as two nontransformed cell lines (WI-38 and Jekin) showed little cell death after a 20-hr AS2 treatment. Since p53 activation can lead to apoptosis or cell cycle arrest, dependent on the level of p53 and the status of the cell, several of these cell lines were further tested for
MCF7-BP100-luc

Fig. 5. Induction of p53 transcriptional activation function. Cells stably transfected with the BP100-luciferase reporter were treated with antisense oligonucleotides at indicated concentrations for $20 \mathrm{hr}$. Luciferase activity/unit protein was determined and the magnitude of induction was shown, compared to cells not treated with oligonucleotides.

AS2M4, a 4-bp mismatch control of AS2; K, an unrelated oligonucleotide. growth arrest by AS2. Cells were treated with AS2 or control oligonucleotides for $20 \mathrm{hr}$, and DNA synthesis was quantitated by BrdU incorporation. The number of viable cells was determined by incubation with the MTS reagent. The results show that in cell lines that do not undergo significant apoptosis after AS2 treatment, the rate of DNA synthesis is reduced (Fig. 7). This effect is weaker with a 4-bp mismatch control oligonucleotide and is not observed with the unrelated oligonucleotide $\mathrm{K}$. In contrast, the p53null cell lines H1299 (human) and 10(1) (mouse) (23) did not undergo significant growth inhibition. Therefore induction of p53 by inhibition of MDM2 can lead to growth arrest or apoptosis.

\section{MDM2-Mediated Degradation of $p 53$ Is Inefficient in Two Choriocarcinoma Cell Lines}

The results presented in Table 1 also reproduce our previous observation that in JAR and JEG-3 cells with high levels of p53 and MDM2, inhibition of MDM2 expression did not result in a significant increase in p53 level. These notable exceptions suggest that the ability of MDM2 to promote p53 degradation may be suppressed in choriocarcinoma cell lines, which predict that p53 is not actively degraded through the ubiquitin-dependent proteasome pathway in these cells.

To test this possibility, JAR and JEG-3 cells were treated with the proteasome inhibitor MG132, which blocks ubiquitin-dependent degradation of proteins (24). PA-1 cells that have highly inducible p53 after MDM2 antisense 
A
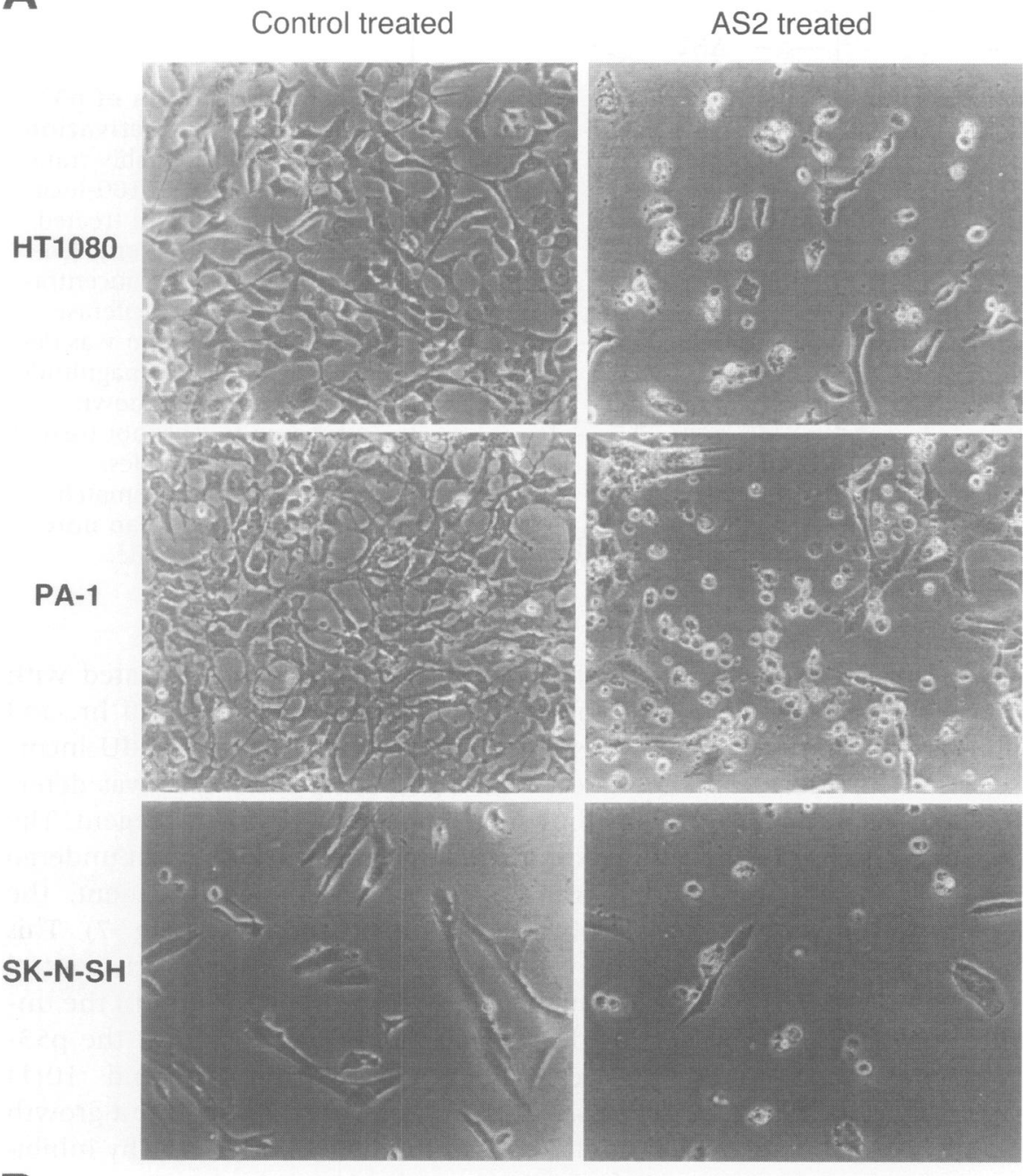

8

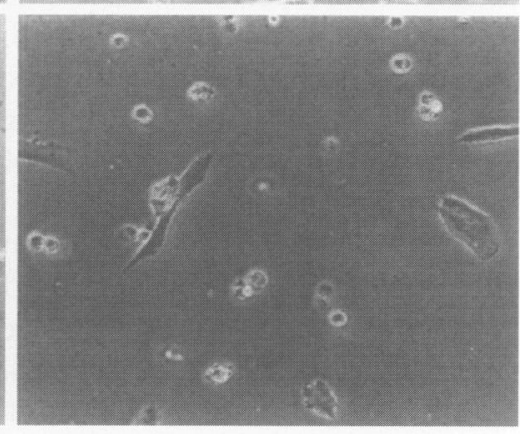

Control treated

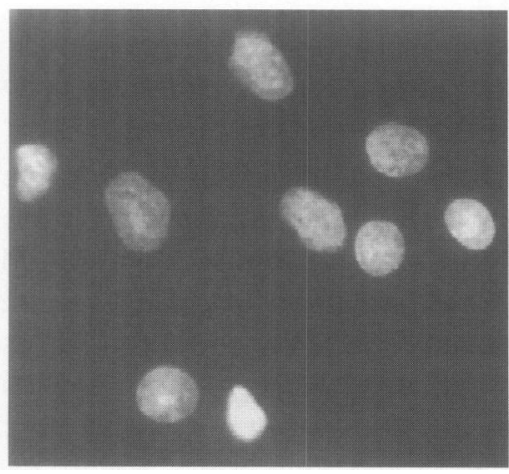

AS2 treated

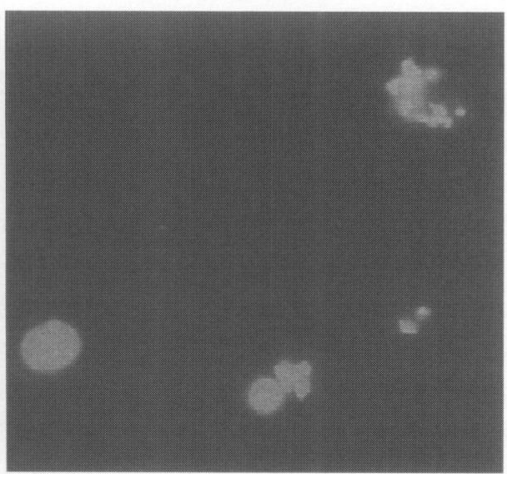

Fig. 6. Induction of cell death by inhibition of MDM2 expression. Cells were treated with $200 \mathrm{nM}$ AS2 or control oligonucleotide $\mathrm{K}$ for $24 \mathrm{hr}$ and photographed. (A) Examples of cell lines undergoing significant cell death characteristic of apoptosis are shown.

(B) SK-N-SH cells treated with AS2 were stained with the DNA dye DAPI to reveal nuclear fragmentation and shrinkage of apoptotic cells. treatment were used as a control. Treatment with $10 \mu \mathrm{M}$ of MGl32 for 3-6 hr resulted in an over 10-fold increase in MDM2 in all three cell lines (Fig. 8), confirming a previous report that MDM2 is degraded through the ubiquitin-dependent proteasome pathway (25). MGl32 only induced a weak ( $<2$-fold) increase of p53 in JAR cells and JEG-3 cells (Fig. 8). In contrast, PA-1 cells showed an over 5 -fold increase in p53 level after MG132 treatment. These results confirm that p53 is not efficiently degraded by the ubiquitin-dependent proteasome pathway in JAR 

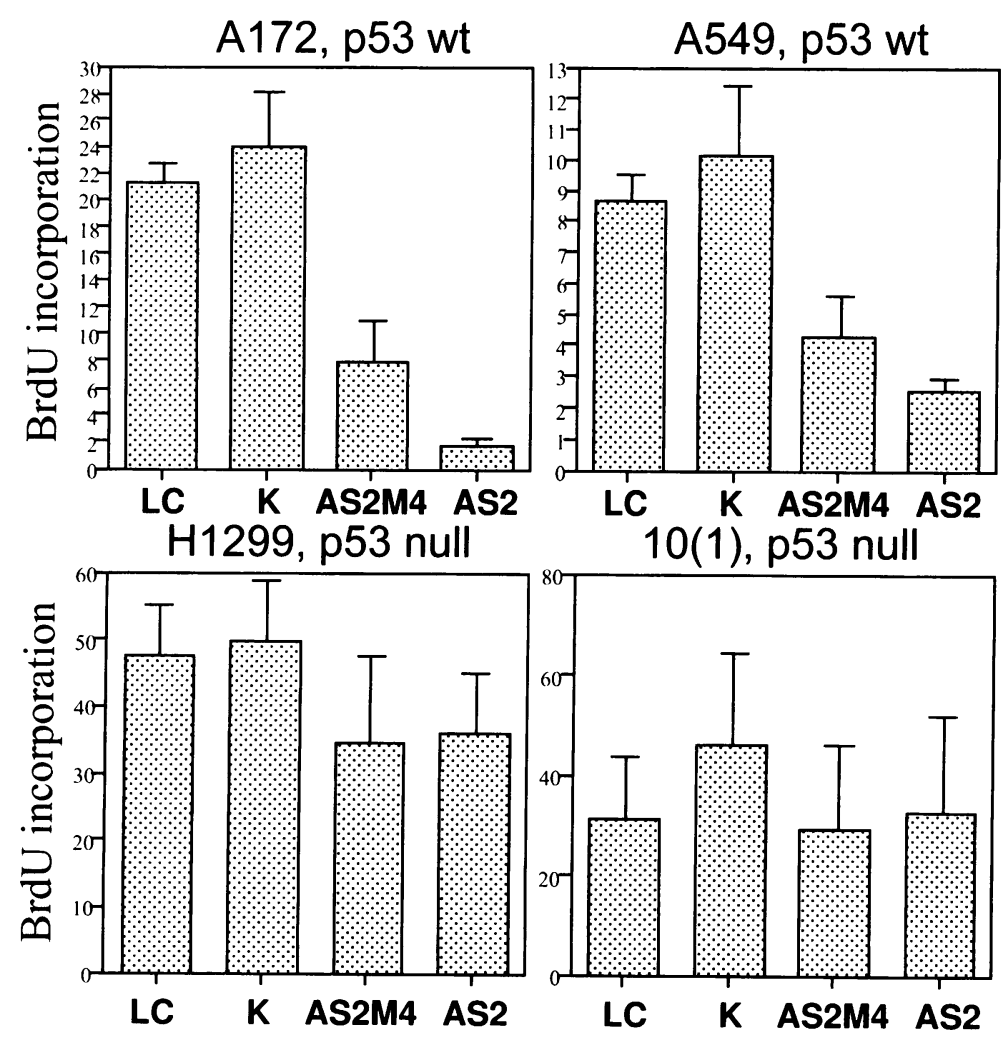

Fig. 7. Inhibition of DNA synthesis by $M D M 2$ antisense oligonucleotides. Cells were treated with $100 \mathrm{nM}$ of oligonucleotides for $20 \mathrm{hr}$ and labeled for $2 \mathrm{hr}$ with BrdU. Incorporation rate of BrdU was determined by an ELISA assay and normalized to the number of viable cells. H1299 and 10(1) cells are human and mouse cells devoid of p53. LC, lipofectin treatment alone.

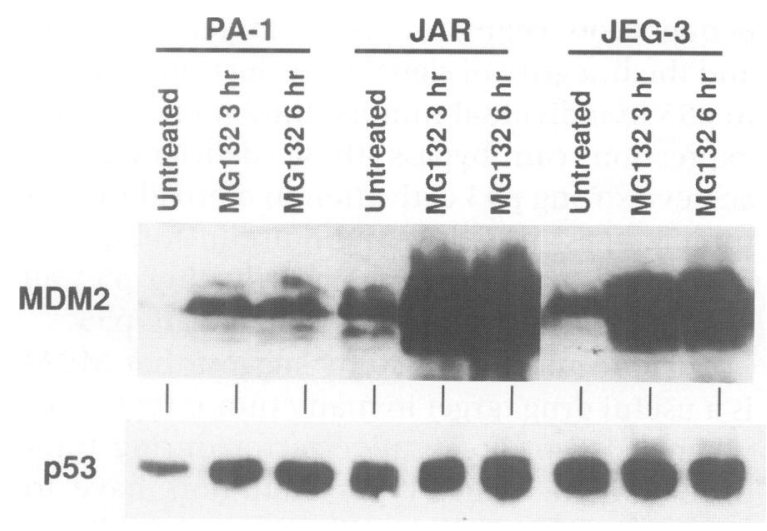

Fig. 8. Effects of proteasome inhibitor MG132 on p53 and MDM2 levels. Cells were treated with $10 \mu \mathrm{M} \mathrm{MGl} 32$ for 3 or $6 \mathrm{hr}$. Identical amounts of total cellular protein were fractionated by SDS-PAGE and analyzed by Western blot with monoclonal antibody 3G9 for MDM2 levels, and DO-1 for p53 levels.

and JEG-3 cells, consistent with the fact that they have stabilized wild-type p53 in the presence of high levels of MDM2 (26).

\section{Discussion}

In this study, we have demonstrated that antisense inhibition of MDM2 expression activates p53 in tumor cells containing either low or high levels of MDM2. Importantly, inhibition of MDM2 expression in cells with low levels of p53 uniformly results in p53 accumulation and increase in p53 activity. This response occurred in nearly all of the tumor and nontransformed cells tested. The only exceptions were HPV-positive cells, which have an E6 oncogene-mediated mechanism of p53 degradation. The accumulation of p53 is due to a prolonged half-life, not an increased rate of synthesis. These results suggest that MDM2 expression is important for maintaining $\mathrm{p} 53$ at low levels. The results also suggest that in addition to possibly playing a role in post-stress down-regulation of $\mathrm{p} 53(9,10,27)$, the p53-degradation function of MDM2 is constitutively active in tumor cells in non-stress conditions.

Induction of DNA strand breaks by a variety of methods have been shown to cause p53 accumulation $(28,29)$, probably by modulation of p53-MDM2 binding (27). The control experiments we performed suggest that MDM2 antisense oligonucleotide induces strong p53 accumulation mainly through a target-specific antisense mechanism. The activation of p53 by AS2 is highly dependent on a perfect sequence match with the target, and correlates with inhi- 
bition of MDM2 expression. A control oligonucleotide with no sequence relationship to MDM2 only weakly activates p53 ( $\sim 2$-fold) at concentrations above $300 \mathrm{nM}$. Therefore, the nonspecific effect of oligonucleotides on $\mathrm{p} 53$ is negligible at the dosages we used.

Our observations suggest that MDM2 overexpression is not the only indicator for $\mathrm{p} 53$ being in a functionally suppressed state. In tumor cells with low levels of MDM2 (which usually correlates with low levels of wild-type p53), MDM2 is also important for inhibiting p53 accumulation. It is possible that the p53-suppression efficiency of the MDM2 feedback loop has increased during tumor development, such as due to p53-independent induction of MDM2 expression (30). Thus, MDM2 can play an active role in tumor development in the absence of overexpression because of imbalance of the p53MDM2 regulatory loop. Alternatively, having low levels of wild-type p53 and MDM2 in a tumor may indicate that the $M D M 2$ regulatory loop is not reacting to tumor development because of defects in the signal transduction pathway, or that certain types of oncogenic changes do not trigger a $\mathrm{p} 53$ response.

Cytoplasmic accumulation of wild-type p53 is frequently observed in breast tumors and neuroblastomas $(21,31)$, and may represent an alternative mechanism of p53 inactivation. A model of cytoplasmic p53 sequestration through inhibition of nuclear translocation has been proposed to explain this phenomenon (32). Our results with MCF-7 and SK-N-SH cells suggest that MDM2 plays an important role in preventing nuclear p53 accumulation in these cells. Inhibition of MDM2 expression can lead to accumulation of nuclear p53 and increase in total cellular p53. Recent findings suggest that MDM2 can promote $\mathrm{p} 53$ degradation $(9,10)$, and this activity is dependent on the nuclear export function of MDM2 (33). Collectively, these results suggest that the cytoplasmic p53 phenotype reflects a dynamic process in which p53 enters the nucleus, binds to MDM2, exits the nucleus as a p53-MDM2 complex, and is degraded or sequestered into cytoplasmic particles. Other experimental approaches are needed to further elucidate this phenomenon.

Two choriocarcinoma cell lines (JAR, JEG-3) show an interesting deviation from most cell lines. Consistent with the observation that they have high levels of MDM2 and stable wild-type p53 (26), inhibition of MDM2 expression or inhibition of proteasome function do not lead to significant accumulation of $p 53$. These results are consistent with the notion that in JAR and JEG-3 cells, MDM2 inhibits p53 mainly through formation of inactive complexes, not promotion of p53 turnover. Whether this is characteristic of trophoblastic tumors and reflects a defect (e.g., lack of a co-factor, point mutation in MDM2) or an active regulation of this degradation pathway remains to be investigated.

Our results demonstrate that inhibition of MDM2 expression is a highly efficient method of activating p53 without causing DNA damage, and may have therapeutic potential against a wide range of tumors. However, systemic inhibition of MDM2 expression will also activate p53 in normal cells that are undergoing rapid proliferation, such as hematopoietic cells and mucosal epithelial cells. These cells are sensitive to p53induced apoptosis after DNA damaging treatments, as indicated by experiments using p53 knockout mice (34-37). Therefore, the anti-tumor specificity and general toxicity of MDM2 inhibitors require further study.

Inhibition of MDM2 activates p53 uniformly and much more efficiently than many DNAdamaging chemotherapy agents (camptothecin, adriamycin, cisplatin, etoposide, fluorouracil, and methotrexate, unpublished results, J. Chen). The ability of these drugs to activate p53 is dependent on cellular uptake, target sensitivity, and the integrity of signal transduction pathways to $\mathrm{p} 53$ in individual tumors. Inhibition of MDM2 expression can bypass these deficiencies and achieve strong p53 activation in a broad range of tumor types; thus it may be useful to augment drugs that are inefficient in activating p53 and complement drugs that do not activate $\mathrm{p} 53$.

The results of this study suggest that MDM2 is a useful drug target in many tumor types, even when it is not a causative factor during tumor development. Many types of tumors have frequencies of $20-30 \%$ (2). Therefore, inhibitors of MDM2 may be useful for the majority of such cases. With the recent approval of the first antisense oligonucleotide-based drug, Fomivirsen, for the treatment of CMV retinitis in AIDS patients, it is evident that this type of compound also has a therapeutic potential. The AS2 oligonucleotide provides a starting point for chemical improvements and further investigation of its anti-tumor potential.

\section{Acknowledgments}

We are particularly grateful to Dr. Jay Hunt for use of the fluorescence microscope. We also 
thank Joelle Finley, Dr. James Gnarra, and Dr. Om Prakash for providing cell lines, and Dr. Ronald Luftig for critical reading of the manuscript. This work was supported by funding from the Stanley S. Scott Cancer Center and a grant from the American Cancer Society to J. Chen.

\section{References}

1. Levine AJ. (1997) p53, the cellular gatekeeper of growth and division. Cell 88: 323-331.

2. Hollstein M, Rice K, Greenblatt MS, et al. (1994) Database of $\mathrm{p} 53$ gene somatic mutations in human tumors and cell lines. Nucl. Acids Res. 22: 35513555.

3. Oliner JD, Kinzler KW, Meltzer PS, George DL, Vogelstein B. (1992) Amplification of a gene encoding a p53-associated protein in human sarcomas. Nature 358: 80-83.

4. Cordon Cardo C, Latres E, Drobnjak M, et al. (1994) Molecular abnormalities of mdm2 and p53 genes in adult soft tissue sarcomas. Cancer Res. 54: 794-799.

5. Lowe SW, Ruley HE, Jacks T, Housman DE. (1993) p53-dependent apoptosis modulates the cytotoxicity of anticancer agents. Cell 74: 957-967.

6. Fakharzadeh SS, Trusko SP, George DL. (1991) Tumorigenic potential associated with enhanced expression of a gene that is amplified in a mouse tumor cell line. EMBO J. 10: 1565-1569.

7. Finlay CA. (1993) The mdm-2 oncogene can overcome wild-type p53 suppression of transformed cell growth. Mol. Cell Biol. 13: 301-306.

8. Momand J, Zambetti GP, Olson DC, George D, Levine AJ. (1992) The mdm-2 oncogene product forms a complex with the $\mathrm{p} 53$ protein and inhibits p53-mediated transactivation. Cell 69: 1237-1245.

9. Haupt Y, Maya R, Kazaz A, Oren M. (1997) Mdm2 promotes the rapid degradation of p53. Nature 387: 296-299.

10. Kubbutat MHG, Jones SN, Vousden KH. (1997) Regulation of p53 stability by mdm2. Nature 387: 299-303.

11. Honda R, Tanaka H, Yasuda H. (1997) Oncoprotein MDM2 is a ubiquitin ligase E3 for tumor suppressor p53. FEBS Lett. 420: 25-27.

12. Wu X, Bayle JH, Olson D, Levine AJ. (1993) The p53-mdm-2 autoregulatory feedback loop. Genes Dev. 7: 1126-1132.

13. Barak Y, Juven T, Haffner R, Oren M. (1993) mdm2 expression is induced by wild type p53 activity. EMBO J. 12: 461-468.

14. Oca Luna RM, Wagner DS, Lozano G. (1995) Rescue of early embryonic lethality in mdm2-deficient mice by deletion of p53. Nature 378: 203206.

15. Jones SN, Roe AE, Donehower LA, Bradley A. (1995) Rescue of embryonic lethality in Mdm2- deficient mice by absence of p53. Nature 378: 206-208.

16. Midgley CA, Lane DP. (1997) p53 protein stability in tumour cells is not determined by mutation but is dependent on $\mathrm{Mdm} 2$ binding. Oncogene 15: 1179-1189.

17. Bottger A, Bottger V, Sparks A, Liu WL, Howard SF, Lane DP. (1997) Design of a synthetic Mdm2binding mini protein that activates the p53 response in vivo. Curr. Biol. 7: 860-869.

18. Chen L, Agrawal S, Zhou W, Zhang R, Chen J. (1998) Synergistic activation of p53 by inhibition of mdm2 expression and DNA damage. Proc. Natl. Acad. Sci. U.S.A. 95: 195-200.

19. Chen J, Marechal V, Levine AJ. (1993) Mapping of the p53 and mdm-2 interaction domains. Mol. Cell Biol. 13: 4107-4114.

20. Takahashi K, Sumimoto H, Suzuki K, Ono T. (1993) Protein synthesis-dependent cytoplasmic translocation of $\mathrm{p} 53$ protein after serum stimulation of growth-arrested MCF-7 cells. Mol. Carcinogen 8: 58-66.

21. Moll UM, LaQuaglia M, Benard J, Riou G. (1995) Wild-type p53 protein undergoes cytoplasmic sequestration in undifferentiated neuroblastomas but not in differentiated tumors. Proc. Natl. Acad. Sci. U.S.A. 92: 4407-4411.

22. Goldman SC, Chen CY, Lansing TJ, Gilmer TM, Kastan MB. (1996) The p53 signal transduction pathway is intact in human neuroblastoma despite cytoplasmic localization. Am. J. Pathol. 148: 1381-1385.

23. Harvey DM, Levine AJ. (1991) p53 alteration is a common event in the spontaneous immortalization of primary BALB/c murine embryo fibroblasts. Genes Dev. 5: 2375-2385.

24. Rock KL, Gramm C, Rothstein L, et al. (1994) Inhibitors of the proteasome block the degradation of most cell proteins and the generation of peptides presented on MHC class I molecules. Cell 78: 761-771.

25. Chang YC, Lee YS, Tejima T, et al. (1998) mdm2 and bax, downstream mediators of the p53 response, are degraded by the ubiquitin-proteasome pathway. Cell Growth Differ. 9: 79-84.

26. Landers JE, Haines DS, Strauss JF, George DL. (1994) Enhanced translation: a novel mechanism of mdm2 oncogene overexpression identified in human tumor cells. Oncogene 9: 2745-2750.

27. Shieh SY, Ikeda M, Taya Y, Prives C. (1997) DNA damage-induced phosphorylation of p53 alleviates inhibition by MDM2. Cell 91: 325-334.

28. Kuerbitz SJ, Plunkett BS, Walsh WV, Kastan MB. (1992) Wild-type p53 is a cell cycle checkpoint determinant following irradiation. Proc. Natl. Acad. Sci. U.S.A. 89: 7491-7495.

29. Nelson WG, Kastan MB. (1994) DNA strand breaks: the DNA template alterations that trigger p53-dependent DNA damage response pathways. Mol. Cell Biol. 14: 1815-1823. 
30. Shaulian E, Resnitzky D, Shifman O, et al. (1997) Induction of Mdm2 and enhancement of cell survival by bFGF. Oncogene 15: 2717-2725.

31. Moll UM, Riou G, Levine AJ. (1992) Two distinct mechanisms alter p53 in breast cancer: mutation and nuclear exclusion. Proc. Natl. Acad. Sci. U.S.A. 89: 7262-7266.

32. Moll UM, Ostermeyer AG, Haladay R, Winkfield B, Frazier M, Zambetti G. (1996) Cytoplasmic sequestration of wild-type p53 protein impairs the Gl checkpoint after DNA damage. Mol. Cell Biol. 16: 1126-1137.

33. Roth J, Dobbelstein M, Freedman DA, Shenk T, Levine AJ. (1998) Nucleo-cytoplasmic shuttling of the hdm2-oncoprotein regulates the levels of $\mathrm{p} 53$ protein via a pathway used by the human immunodeficiency virus rev protein. EMBO J. 17: 554564.

34. Clarke AR, Gledhill S, Hooper ML, Bird CC, Wyllie
AH. (1994) p53 dependence of early apoptotic and proliferative responses within the mouse intestinal epithelium following gamma-irradiation. Oncogene 9: 1767-1773.

35. Lowe SW, Schmitt EM, Smith SW, Osborne BA, Jacks T. (1993) p53 is required for radiation-induced apoptosis in mouse thymocytes. Nature 362: 847-849.

36. Gottlieb E, Haffner R, King A, et al. (1997) Transgenic mouse model for studying the transcriptional activity of the $\mathrm{p} 53$ protein: age- and tissue-dependent changes in radiation-induced activation during embryogenesis. EMBO J. 16: 1381-1390.

37. Komarova EA, Chernov MV, Franks R, et al. (1997) Transgenic mice with p53-responsive lacZ: p53 activity varies dramatically during normal development and determines radiation and drug sensitivity in vivo. EMBO J. 16: 1391-1400. 